\title{
The gas filtration in complex porous media with stagnant zones
}

\author{
Pyanylo Ya. D., Bratash O. B. \\ Lviv Center of Mathematical Modeling of Pidstryhach Institute \\ for Applied Problems of Mechanics and Mathematics, \\ National Academy of Sciences of Ukraine, \\ 15 Dudayev Str., 79005, Lviv, Ukraine
}

(Received 1 April 2019; Accepted 5 May 2020)

\begin{abstract}
The process of gas filtration in a porous medium depending on its structure is modeled in the paper. The presence of pores of various sizes leads to the formation of flow and stagnation zones, which affect both the pressure distribution in the medium and the active gas mass. The obtained results make it possible to determine the proportion of the flow zones volume and the exchange coefficient between the flow and stagnant zones.
\end{abstract}

Keywords: gas filtration, porous medium, partial differential equations, integral transforms.

2010 MSC: $93 \mathrm{C} 23,49 \mathrm{~K} 20$

DOI: $10.23939 / \mathrm{mmc} 2020.01 .179$

\section{Introduction}

Natural liquids such as oil, gas, underground water are located in the Earth subsoils, or rather, in underground cavities - pores and cracks of the rocks [1]. Underground gas storages (UGS) are mainly formed in depleted gas, aquiferous layers, gas condensate and oil fields [2,3]. When creating UGS preference is given to the places where depleted gas fields which have a pore volume sufficient to store the required active gas volume, satisfactory filtration-capacitive properties and relatively homogeneous distribution of them in the area and section of the layer-collector are located. In the process of creating UGS, issues of the rational ratio of buffer and active gas, number of wells, maximum and minimum pressures in the layer-collector, compressor station power, etc. should be solved.

The structure of the rocks pore space is caused by granulometric composition of particles, their shape, the chemical composition of the rocks, the origin of pores, as well as the ratio of the number of large and small pores. The structure of the rock is predominantly determined by the size and shape of its grains. By size the structures are distinguished by the following: psephitic (the rock consists of the fragments in the size of more than $1-2 \mathrm{~mm})$, psammitic $(0.1-1 \mathrm{~mm})$, aleurolitic $(0.01-0.1 \mathrm{~mm})$, pelitic $(0.01 \mathrm{~mm}$ and less). To the texture features of the rock are referred layering, nature of the placement and location of the rocks, the location and the quantitative ratio of cement and grains of the rock, as well as some other features of the structure [2-4]. The role of the cement often perform clay substances. There are also cements of chemogenic origin (carbonates, oxides and hydroxides, sulfates). To a greater extent, the properties of porous media depend on the size of the pore channels. According to the size the pore channels of oil and gas layers are conventionally divided into three groups: overcapillary more than $0.5 \mathrm{~mm}$; capillary - from 0.5 to $0.0002 \mathrm{~mm}(0.2 \mu \mathrm{m})$; sub-capillary - less than $0.0002 \mathrm{~mm}$ $(0.2 \mu \mathrm{m})$. In large (overcapillary) channels and pores, the movement of oil, water and gas passes freely, and in capillary - with the significant participation of capillary forces. In sub-capillary channels, the substances are kept to such an extent by the attraction force of channel walls (due to the small distance between the walls of the channel, the fluid in it is in the sphere of the action of the molecular forces of the rock material), that practically can not move in them under the natural conditions. The average radius $r$ (in $\mathrm{m}$ ) of the pores in the rock exemplar depends on the coefficients of permeability $k$ (in $\mathrm{m}^{2}$ ) and the porosity $\mathrm{m}$ (non-dimensional value) of the rock and is characterized by Slichter number. 
A Slichter number is the non-dimensional function $\operatorname{Sl}(m, \varepsilon)$ of the porosity $m$ and the structure of the porous medium $\varepsilon$, which qualitatively characterizes the coefficient of the permeability $k: k=$ $d_{\mathrm{ef}} \mathrm{Sl}(m, \varepsilon)$, where $d_{\mathrm{ef}}(m)$ is the effective diameter of the particles of the porous medium. When filtration is in a fictitious soil, the Slighter number is a function of porosity, and in the case of filtration in a real soil, in addition, a function of the particles form and the degree of roughness of their surface.

Since in the sub-capillary channels carbohydrates cannot move, then the so-called stagnant zones, which contain carbohydrates, are formed. It is obvious that such stagnant zones are volatile and depend on the pressure in the porous medium. Under certain ratios between layer pressure and pressure in the stagnant zones, carbohydrates from the latter can be released. This, in turn, affects the process of hydrocarbons mining.

The purpose of the work is to construct a mathematical model for calculating the gas flow process in the flow and stagnant zones and to determine the impact of the gas which is located in stagnant zones on its total mining.

\section{Formulation of the problem}

We will consider the process of gas filtration in the complex porous medium, which occupies area of the rectangular parallelepiped of the small thickness. Since the thickness of the medium is small, then the pressure drop in the vertical direction can be neglected and the filtration process can be considered only in the flat case. The process of gas filtration in a complex porous medium with stagnant zones can be described by the following linearized system of differential equations in partial derivatives [5-8]

$$
\left\{\begin{array}{l}
(1-\nu) \frac{\partial p_{2}}{\partial t}+\nu \frac{\partial p_{1}}{\partial t}-a\left(\frac{\partial^{2} p_{1}}{\partial x^{2}}+\frac{\partial^{2} p_{1}}{\partial y^{2}}\right)=0 \\
\frac{\partial p_{2}}{\partial t}=\gamma\left(p_{1}-p_{2}\right)
\end{array}\right.
$$

Here $p_{1}(x, y, t)$ and $p_{2}(x, y, t)$ are the gas pressures in the drain and stagnant zones, $\nu$ is the share of the volume of drain zones, $\gamma$ is the exchange coefficient between zones, $a=2 a_{0} p_{10}, a_{0}=k / \mu \beta$ is the piezoconductance coefficient, $k$ is the coefficient of porous medium permeability, $\mu$ is the dynamic coefficient of viscosity of a liquid, $\beta$ is the coefficient of volumetric elasticity of saturated porous medium, $p_{10}$ is the pressure value defined at the previous step, $x \in\left[x_{0}, x_{k}\right], y \in\left[y_{0}, y_{k}\right]$. As the boundary conditions we will consider conditions under which there is no gas outlet. The boundary value problem is solved for an arbitrary initial condition, which can be set in the process of calculating the pressure distribution in the medium.

\section{Solving the formulated problem}

Let use the Laplace-Carson integral transform. Since

$$
f^{\prime}(t) \Leftrightarrow s[F(s)-f(0)],
$$

then in Laplace-Carson images $[9,10]$ the system (1) will look like

$$
\left\{\begin{array}{l}
(1-\nu) s\left[P_{2}(x, y, s)-p_{2}(x, y, 0)\right]+\nu s\left[P_{1}(x, y, p)-p_{1}(x, y, 0)\right]-a\left(P_{1 x}^{\prime \prime}(x, y, s)+P_{1 y}^{\prime \prime}(x, y, s)\right)=0, \\
s\left[P_{2}(x, y, s)-p_{2}(x, y, 0)\right]=\gamma\left[P_{1}(x, y, s)-P_{2}(x, y, s)\right] .
\end{array}\right.
$$

Here $P_{i}(x, y, s), i=1,2$ is Laplace-Carson image of the function $p_{i}(x, y, s), i=1,2, s$ is the LaplaceCarson transform parameter. From the second equation of the last system we will obtain

$$
P_{2}(x, y, s)=\frac{\gamma}{s+\gamma} P_{1}(x, y, s)+\frac{s}{s+\gamma} p_{2}(x, y, 0) .
$$


To determine the image $P_{1}(x, y, s)$ we will obtain an ordinary differential equation of the second order

$$
\begin{aligned}
{\left[\frac{\gamma(1-\nu) s}{s+\gamma}+\nu s\right] P_{1}(x, y, s)-a } & \left(P^{\prime \prime}{ }_{1 x}(x, y, s)+P^{\prime \prime}{ }_{1 y}(x, y, s)\right) \\
& =(1-\nu) s p_{2}(x, y, 0)+\nu s p_{1}(x, y, 0)-\frac{(1-\nu) s^{2}}{s+\gamma} p_{2}(x, y, 0) .
\end{aligned}
$$

If we introduce the notation

$$
b=\frac{\gamma(1-\nu) s}{s+\gamma}+\nu s, \quad d=(1-\nu) s p_{2}(x, y, 0)+\nu s p_{1}(x, y, 0)-\frac{(1-\nu) s^{2}}{s+\gamma} p_{2}(x, y, 0),
$$

then the equation (3) will look like

$$
P_{1 x}^{\prime \prime}(x, y, s)+P_{1 y}^{\prime \prime}(x, y, s)-\alpha P_{1}(x, y, p)=\beta, \quad \alpha=\frac{b}{a}, \quad \beta=-\frac{d}{a} .
$$

Since the boundary conditions are constant, it is expedient to find the solution of the equation (4) in the the form

$$
P_{1}(x, y, s)=\sum_{n=1}^{\infty} P_{1 n}(y, s) \cos \frac{n \pi\left(x-x_{0}\right)}{x_{k}-x_{0}}
$$

with unknown coefficients

$$
P_{1 n}(y, s)=\frac{2}{x_{k}-x_{0}} \int_{x_{0}}^{x_{k}} P_{1}(x, y, s) \cos \frac{n \pi\left(x-x_{0}\right)}{x_{k}-x_{0}} d x .
$$

Integrating the last equation by parts, taking into account the conditions of impenetrability, leads to the following equation

$$
P_{1 n}(y, s)=\frac{2\left(x_{k}-x_{0}\right)}{(n \pi)^{2}} \int_{x_{0}}^{x_{k}} \frac{d^{2} P_{1}(x, y, s)}{d x^{2}} \cos \frac{n \pi\left(x-x_{0}\right)}{x_{k}-x_{0}} d x .
$$

Since

$$
P^{\prime \prime}{ }_{1 x}(x, y, s)=\beta+\alpha P_{1}(x, y, p)-P_{1 y}^{\prime \prime}(x, y, s),
$$

then

$$
P_{1 n}(y, s)=\frac{2\left(x_{k}-x_{0}\right)}{(n \pi)^{2}} \int_{x_{0}}^{x_{k}}\left(\beta+\alpha P_{1}(x, y, p)-P_{1 y}^{\prime \prime}(x, y, s)\right) \cos \frac{n \pi\left(x-x_{0}\right)}{x_{k}-x_{0}} d x
$$

from which we obtain the differential equation for determining unknown coefficients

$$
P_{1 n}(y, s)=\frac{\left(x_{k}-x_{0}\right)^{2}}{(n \pi)^{2}}\left\{\alpha P_{1 n}(y, s)-P^{\prime \prime}{ }_{1 n y}(y, s)\right\}
$$

or

$$
P_{1 n y}^{\prime \prime}(y, s)-\left[\alpha+\left(\frac{n \pi}{x_{k}-x_{0}}\right)^{2}\right] P_{1 n}(y, s)=0 .
$$

If we will mark $\lambda_{n}^{2}=\alpha+\left(\frac{n \pi}{x_{k}-x_{0}}\right)^{2}$, then as a characteristic equation for an ordinary homogeneous differential equation

$$
P_{1 n y}^{\prime \prime}(y, s)-\lambda_{n}^{2} P_{1 n}(y, s)=0
$$

is an algebraic equation $z^{2}-\lambda_{n}^{2}=0$, the roots of which are defined as follows $z_{1}=\lambda_{n}, z_{2}=-\lambda_{n}$. Then the solution of the equation will have the form

$$
P_{1 n}(y, s)=A(s) e^{y \lambda_{n}}+B(s) e^{-y \lambda_{n}} .
$$


Let us suppose, for simplicity, $P_{1}(x, 0, t)=\theta_{1 y}(0) \equiv$ const. Then $P_{1}(x, 0, s)=\theta_{1 y}(0) \equiv$ const and for determining $A(s)$ and $B(s)$ the following system is obtained

$$
\begin{gathered}
A(s)+B(s)=2 \theta_{1 y}, \\
A(s) e^{y_{0} \lambda_{n}}+B(s) e^{-y_{0} \lambda_{n}}=2 \theta_{1 y},
\end{gathered}
$$

the solution of which has the form

$$
A(s)=2 \theta_{1 y} \frac{e^{-y_{0} \lambda_{n}}-1}{e^{-y_{0} \lambda_{n}}-e^{y_{0} \lambda_{n}}}, \quad B(s)=2 \theta_{1 y} \frac{1-e^{y_{0} \lambda_{n}}}{e^{-y_{0} \lambda_{n}}-e^{y_{0} \lambda_{n}}} .
$$

Then in Laplace-Carson space

$$
P_{1 n}(y, s)=\frac{2 \theta_{1 y}}{\sinh \left(y_{0} \lambda_{n}\right)}\left[\sinh \left(y \lambda_{n}\right)+\sinh \left(\left(y_{0}-y\right) \lambda_{n}\right)\right] .
$$

To find unknown coefficients $P_{1 n}(y, t)$, it is necessary to move from the images to the originals in the last formula. To do this, we expand the function

$$
\bar{\varphi}(s, y)=\frac{\sinh \left(y \lambda_{n}\right)}{\sinh \left(y_{0} \lambda_{n}\right)}
$$

in simple fractions. We will obtain

$$
\bar{\varphi}(s, y)=\frac{\sinh \left(y \lambda_{n}\right)}{\sinh \left(y_{0} \lambda_{n}\right)}=\frac{y}{y_{0}}+\frac{2}{\pi} \sum_{k=1}^{\infty} \frac{(-1)^{k}}{k} \frac{\lambda_{n}^{2}}{\lambda_{n}^{2}+\vartheta_{k}} \sin \frac{k \pi y}{y_{0}},
$$

where $\vartheta_{k}=\left(k \pi / y_{0}\right)^{2}$.

Since

$$
\lambda_{n}=\sqrt{\alpha+\left(\frac{n \pi}{x_{k}-x_{0}}\right)^{2}}, \quad \alpha=\frac{\gamma(1-\nu) s}{a(s+\gamma)}+\frac{\nu s}{a},
$$

then

$$
\frac{\lambda_{n}^{2}}{\lambda_{n}^{2}+\vartheta_{k}}=\frac{s^{2}+c_{1} s+c_{2}}{s^{2}+z_{1} s+z_{2}}
$$

where is marked

$$
\begin{gathered}
c_{1}=\frac{\gamma_{1}+v_{1} \gamma+\pi_{n}}{v_{1}}, \quad c_{2}=\frac{\pi_{n} \gamma}{v_{1}}, \quad z_{1}=\frac{\gamma_{1}+v_{1} \gamma+\pi_{n}+\vartheta_{k}}{v_{1}}, \quad z_{2}=\frac{\left(\pi_{n}+\vartheta_{k}\right) \gamma}{v_{1}}, \\
\gamma_{1}=\frac{\gamma(1-\nu)}{a}, \quad v_{1}=\frac{\nu}{a}, \quad \pi_{n}=\left(\frac{n \pi}{x_{k}-x_{0}}\right)^{2} .
\end{gathered}
$$

Then

$$
\bar{\varphi}(s, y)=\frac{y}{y_{0}}+\frac{2}{\pi} \sum_{k=1}^{\infty} \frac{(-1)^{k}}{k} \frac{s^{2}+c_{1} s+c_{2}}{\left(s-s_{1}\right)\left(s-s_{2}\right)} \sin \frac{k \pi y}{y_{0}},
$$

where

$$
s_{1,2}=-\frac{z_{1}}{2} \pm \sqrt{\left(\frac{z_{1}}{2}\right)^{2}-z_{2}} .
$$

There is operational equality [9]

$$
\frac{s^{2}+c_{1} s+c_{2}}{\left(s-s_{1}\right)\left(s-s_{2}\right)} \div \frac{c_{1}}{s_{1} s_{2}}+\frac{s_{1}^{2}+c_{1} s_{1}+c_{2}}{-s_{1}\left(-s_{1}-s_{2}\right)} e^{s_{1} t}-\frac{s_{2}^{2}+c_{1} s_{2}+c_{2}}{-s_{2}\left(-s_{1}-s_{2}\right)} e^{s_{2} t}=\psi_{k}(t) .
$$

Mathematical Modeling and Computing, Vol. 7, No. 1, pp. 179-185 (2020) 
Then

$$
\varphi(t, y)=\frac{y}{y_{0}}+\frac{2}{\pi} \sum_{k=1}^{\infty} \frac{(-1)^{k}}{k} \psi_{k}(t) \sin \frac{k \pi y}{y_{0}}
$$

and

$$
P_{1 n}(y, t)=2 \theta_{1 y}\left[\varphi(t, y)+\varphi\left(t, y_{0}-y\right)\right] .
$$

Thus, the final pressure distribution in the flow zone will look like

$$
P_{1}(x, y, t)=\sum_{n=1}^{\infty} P_{1 n}(y, t) \cos \frac{n \pi\left(x-x_{0}\right)}{x_{k}-x_{0}} .
$$

Let us find the pressure distribution in stagnant zones. Since

$$
P_{2}(x, y, s)=\frac{\gamma}{s+\gamma} P_{1}(x, y, s)+\frac{s}{s+\gamma} p_{2}(x, y, 0)
$$

and

$$
\frac{1}{s+\gamma} \div \frac{1}{\gamma}\left(1-e^{-\gamma t}\right), \quad \frac{s}{s+\gamma} \div e^{-\gamma t},
$$

then, using a convolution theorem can be written

$$
\begin{aligned}
P_{2}(x, y, t) & =\frac{d}{d t} \int_{0}^{t}\left(1-e^{-\gamma(t-\tau)}\right) P_{1}(x, y, \tau) d \tau+e^{-\gamma t} p_{2}(x, y, 0) \\
& =\gamma \int_{0}^{t} e^{-\gamma(t-\tau)} P_{1}(x, y, \tau) d \tau+e^{-\gamma t} p_{2}(x, y, 0) .
\end{aligned}
$$

Let us introduce the notations

$$
\zeta_{0}=\frac{c_{1}}{s_{1} s_{2}}, \quad \zeta_{1}=\frac{s_{1}^{2}+c_{1} s_{1}+c_{2}}{-s_{1}\left(-s_{1}-s_{2}\right)}, \quad \zeta_{2}=-\frac{s_{2}^{2}+c_{1} s_{2}+c_{2}}{-s_{2}\left(-s_{1}-s_{2}\right)} .
$$

Then

$$
\psi_{k}(t)=\zeta_{0}+\zeta_{1} e^{s_{1} t}+\zeta_{2} e^{s_{2} t}
$$

Next, let us present the integral in the formula (5) as follows

$$
\int_{0}^{t} e^{-\gamma(t-\tau)} \sum_{n=1}^{\infty} P_{1 n}(y, \tau) \cos \frac{n \pi\left(x-x_{0}\right)}{x_{k}-x_{0}} d \tau=\sum_{n=1}^{\infty} \cos \frac{n \pi\left(x-x_{0}\right)}{x_{k}-x_{0}} \int_{0}^{t} e^{-\gamma(t-\tau)} P_{1 n}(y, \tau) d \tau .
$$

Let

$$
\operatorname{In}_{1 n}=\int_{0}^{t} e^{-\gamma(t-\tau)} P_{1 n}(y, \tau) d \tau=2 \theta_{1 y} \int_{0}^{t} e^{-\gamma(t-\tau)}\left[\varphi(\tau, y)+\varphi\left(\tau, y_{0}-y\right)\right] d \tau
$$

Then

$$
\begin{aligned}
\operatorname{In}_{1 n \varphi}(y, t) & =\int_{0}^{t} e^{-\gamma(t-\tau)}\left[\frac{y}{y_{0}}+\frac{2}{\pi} \sum_{k=1}^{\infty} \frac{(-1)^{k}}{k} \psi_{k}(\tau) \sin \frac{k \pi y}{y_{0}}\right] d \tau \\
& =\frac{y}{y_{0}} \int_{0}^{t} e^{-\gamma(t-\tau)} d \tau+\frac{2}{\pi} \sum_{k=1}^{\infty} \frac{(-1)^{k}}{k} \sin \frac{k \pi y}{y_{0}} \int_{0}^{t} e^{-\gamma(t-\tau)} \psi_{k}(\tau) d \tau
\end{aligned}
$$

Since

$$
w_{k}=\int_{0}^{t} e^{-\gamma(t-\tau)} \psi_{k}(\tau) d \tau=\int_{0}^{t} e^{-\gamma(t-\tau)}\left[\zeta_{0}+\zeta_{1} e^{s_{1} \tau}+\zeta_{2} e^{s_{2} \tau}\right] d \tau
$$

Mathematical Modeling and Computing, Vol. 7, No. 1, pp.179-185 (2020) 


$$
=\frac{\zeta_{0}}{\gamma}\left(1-e^{-\gamma t}\right)+\frac{\zeta_{1}}{\gamma+s_{1}}\left(e^{s_{1} t}-e^{-\gamma t}\right)+\frac{\zeta_{2}}{\gamma+s_{2}}\left(e^{s_{2} t}-e^{-\gamma t}\right),
$$

then

$$
\operatorname{In}_{1 n \varphi}(y, t)=\left\{\frac{y}{\gamma y_{0}}\left(1-e^{-\gamma t}\right)+\frac{2}{\pi} \sum_{k=1}^{\infty} \frac{(-1)^{k}}{k} \sin \frac{k \pi y}{y_{0}} w_{k}\right\}
$$

and

$$
I n_{1 n}=\int_{0}^{t} e^{-\gamma(t-\tau)} P_{1 n}(y, \tau) d \tau=2 \theta_{1 y}\left[\operatorname{In}_{1 n \varphi}(y, t)+\operatorname{In}_{1 n \varphi}\left(y_{0}-y, t\right)\right] .
$$

So

$$
F(x, y, t)=\int_{0}^{t} e^{-\gamma(t-\tau)} \sum_{n=1}^{\infty} P_{1 n}(y, \tau) \cos \frac{n \pi\left(x-x_{0}\right)}{x_{k}-x_{0}} d \tau=\sum_{n=1}^{\infty} \cos \frac{n \pi\left(x-x_{0}\right)}{x_{k}-x_{0}} I_{1 n}
$$

and finally we get the pressure distribution in the stagnant zones

$$
P_{2}(x, y, t)=\gamma F(x, y, t)+e^{-\gamma t} p_{2}(x, y, 0) .
$$

\section{Algorithm for determining the parameters of stagnant zones}

The gas mass in the porous medium is calculated by the formula

$$
M=m \int_{V} \rho d V
$$

where $m$ is the medium porosity. If consider a rectangular parallelepiped of thickness $h$, average the pressure by thickness and use the gas law, then the gas mass which present in the flowing pores is calculated by the formula

$$
M_{p}(t)=m h v \int_{y_{0}}^{y_{k}} d y \int_{x_{0}}^{x_{k}} \frac{p_{1}(x, y, t)}{z R T} d x
$$

and the gas mass in stagnant zones

$$
M_{z}(t)=m h(1-v) \int_{y_{0}}^{y_{k}} d y \int_{x_{0}}^{x_{k}} \frac{p_{2}(x, y, t)}{z R T} d x .
$$

The difference of the gas mass in the flow pores over the time $\Delta t=t_{2}-t_{1}, t \in\left[t_{1}, t_{2}\right]$ is calculated by the formula

$$
\Delta M_{p}(\Delta t)=M_{p}\left(t_{2}\right)-M_{p}\left(t_{1}\right)=m h v \int_{y_{0}}^{y_{k}} d y \int_{x_{0}}^{x_{k}} \frac{p_{1}\left(x, y, t_{2}\right)-p_{1}\left(x, y, t_{1}\right)}{z R T} d x .
$$

Experimental determining the share of the flow zones volume is as follows.

1. By the meter devices the mass of gas that was removed from the storage is determined.

2. The mathematical model of the gas diffusion process in the storage layer is constructed and adapted.

3. According to the constructed model, the value $\Delta M_{p}(t)$ is calculated.

4. If the measured mass of gas coincides with the calculated according to the last formula with given accuracy, then the stagnant zones don't participate in the process of gas removal. Otherwise, the share of stagnant zones is determined.

Stagnant zones are characterized by a share of the volume of the flow zones $\nu$ and the exchange coefficient between zones $\gamma$. It is obvious that the determination of these parameters essentially depends on the proportion of stagnant zones, and on the accuracy of the input information and is changed depending on the gas pressure in the UGS layer. If the proportion of stagnant zones in the process of working UGS is beyond the limit of the accuracy of the model, it is not expedient to determine these 
parameters. Otherwise, it is necessary to have some additional information to determine the parameters of the stagnant zones, in particular, the difference between the actually selected and calculated gas volumes.

[1] Prytula N. M., Pyanylo Ya. D., Prytula M. H. Underground storage of gas (mathematical models and methods). Lviv, RASTR-7 (2015), (in Ukrainian).

[2] Leibenzon L. S. The movement of natural liquids and gases in a porous medium. Moscow-Leningrad, State publishing house of technical and theoretical literature (1947), (in Russian).

[3] Barrenblatt H. I., Entov V. M., Ryzhyk V. M. Theory of non-stationary filtration of liquid and gas. Moscow, Nedra (1972), (in Russian).

[4] Varhaftik N. B. Handbook of thermophysical properties of gases and liquids. Moscow, Nauka (1972), (in Russian).

[5] Babenko Yu. I. The method of fractional differentiation in applied problems of the theory of heat and mass transfer. SPb., NPO "Professional" (2009), (in Russian).

[6] Holubev V.S. Equations of motion of a fluid in a porous medium with flow and stagnant zones. Report of Academy of Sciences of USSR. 238 (6), 1318-1320 (1978), (in Russian).

[7] Dietrich P., et al. Flowand Transportin Fractured Porous Media. Springer-Verlag, Berlin (2005).

[8] Bear J. Dynamics of fluids in porous media. New York, American Elsevier publ. (1967).

[9] Ditkin V. A., Prudnikov A. P. Handbook of operational calculus. Moscow, High School (1965), (in Russian).

[10] Ditkin V. A., Prudnikov A. P. Operational calculus. Moscow, High School (1975), (in Russian).

\title{
Фільтрація газу в складному пористому середовищі із застійними зонами
}

\author{
П'янило Я. Д., Браташ О. Б. \\ Центр математичного моделювання \\ Інституту прикладних проблем механіки і математики ім. Я. С. Підстригача НАН Украӥни, \\ вул. Дж. Дудаєва, 15, 79005, Лъвів, Україна
}

У роботі моделюється процес фільтрації газу в пористому середовищі залежно від його структури. Наявність пор різних розмірів зумовлюе утворення протічних і застійних зон, які впливають як на розподіл тиску в середовищі, так і на масу активного газу. Отримані результати дають можливість визначити частку об'єму протічних зон та коефіцієнт обміну між протічними та застійними зонами.

Ключові слова: фільтрачія газу, пористе середовище, диференціальні рівняння в частинних похідних, інтегральні перетворення. 